\title{
Decomposition and stability of linear singularly perturbed systems with two small parameters
}

\author{
Osypova O.V., Pertsov A.S., Cherevko I.M.
}

In the domain $\Omega=\left\{\left(t, \varepsilon_{1}, \varepsilon_{2}\right): t \in \mathbb{R}, \varepsilon_{1}>0, \varepsilon_{2}>0\right\}$, we consider a linear singularly perturbed system with two small parameters

$$
\left\{\begin{array}{l}
\dot{x}_{0}=A_{00} x_{0}+A_{01} x_{1}+A_{02} x_{2} \\
\varepsilon_{1} \dot{x}_{1}=A_{10} x_{0}+A_{11} x_{1}+A_{12} x_{2} \\
\varepsilon_{1} \varepsilon_{2} \dot{x}_{2}=A_{20} x_{0}+A_{21} x_{1}+A_{22} x_{2}
\end{array}\right.
$$

where $x_{0} \in \mathbb{R}^{n_{0}}, x_{1} \in \mathbb{R}^{n_{1}}, x_{2} \in \mathbb{R}^{n_{2}}$. In this paper, schemes of decomposition and splitting of the system into independent subsystems by using the integral manifolds method of fast and slow variables are investigated. We give the conditions under which the reduction principle is truthful to study the stability of zero solution of the original system.

Key words and phrases: singularly perturbed system, decomposition, splitting, stability, integral manifold.

\footnotetext{
Yuriy Fedkovych Chernivtsi National University, 2 Kotsjubynskyi str., 58012, Chernivtsi, Ukraine

E-mail: shurenkacv@gmail.com (Osypova O.V.), pertsov@ukr.net (Pertsov A.S.),

i. cherevko@chnu.edu.ua (Cherevko I.M.)
}

\section{Introduction}

The theory of singularly perturbed systems of differential equations has been intensively developed recently, and its methods have been widely used for the study of various problems of science and technique. Singularly perturbed models describe dynamic processes in physics, chemical kinetics, theory of automatic control, hydroscopic systems, economical and biological processes etc.

For singularly perturbed systems, an important task is to develop asymptotic methods for investigating the qualitative behavior of both individual solutions and their sets. Effective method of investigation of singularly perturbed systems is integral manifold method [4,11], which allows to reduce the dimension of the initial system on the integral manifold. In many applications of singularly perturbed systems an important role is played by the transformation of variables, which makes it possible to decompose the initial system to a block-triangular form [7]. In particular, algorithms for constructing asymptotic decompositions of an integral manifold by degrees of a small parameter, that can be effectively implemented in systems of computer algebra, are investigated in $[3,6]$.

The application of the integral manifold method to the decomposition and splitting of linear singularly perturbed systems into independent fast and slow subsystems proved to be 
interesting and effective $[2,9,10]$. In particular, for a linear system

$$
\left\{\begin{array}{l}
\dot{x}=A_{11} x+A_{12} y \\
\varepsilon \dot{y}=A_{21} x+A_{22} y
\end{array}\right.
$$

were established the conditions for existence of variables substitution

$$
\left\{\begin{array}{l}
x=u+\varepsilon P(t, \varepsilon) v \\
y=v+H(t, \varepsilon) x
\end{array}\right.
$$

which transforms the initial system into two independent subsystems

$$
\begin{aligned}
& \dot{u}=\left(A_{11}+A_{12} H\right) u, \\
& \varepsilon \dot{v}=\left(A_{22}-\varepsilon H A_{12}\right) v .
\end{aligned}
$$

The application of the method of integral manifolds of fast and slow variables to the studying of linear singularly perturbed systems with many small parameters was carried out in $[1,5,8,12]$. In this paper, we establish the reduction principle for investigation the stability of linear singularly perturbed systems with two small parameters.

\section{Decomposition}

In the domain $\Omega=\left\{\left(t, \varepsilon_{1}, \varepsilon_{2}\right): t \in \mathbb{R}, \varepsilon_{1}>0, \varepsilon_{2}>0\right\}$, we consider a linear singularly perturbed system

$$
\left\{\begin{array}{l}
\dot{x}_{0}=A_{00} x_{0}+A_{01} x_{1}+A_{02} x_{2}, \\
\varepsilon_{1} \dot{x}_{1}=A_{10} x_{0}+A_{11} x_{1}+A_{12} x_{2}, \\
\varepsilon_{1} \varepsilon_{2} \dot{x}_{2}=A_{20} x_{0}+A_{21} x_{1}+A_{22} x_{2}
\end{array}\right.
$$

where $x_{0} \in \mathbb{R}^{n_{0}}, x_{1} \in \mathbb{R}^{n_{1}}, x_{2} \in \mathbb{R}^{n_{2}}, A_{i j}=A_{i j}(t), i, j=\overline{0,2}$, are $n_{i} \times n_{j}$ matrices, and $\varepsilon_{1}, \varepsilon_{2}$ are small positive parameters.

Let the following conditions hold:

C.1) matrices $A_{i j}(t), i, j=\overline{0,2}$, are uniformly bounded in $t \in \mathbb{R}$ by a positive constant $M$;

C.2) eigenvalues $\lambda_{i}=\lambda_{i}(t), i=\overline{1, n_{2}}$, of the matrix $A_{22}(t)$ satisfy the inequality

$$
\operatorname{Re} \lambda_{i} \leq-2 \beta<0 \text {. }
$$

Let us make in (1) the substitution

$$
x_{0}=y_{0}+\varepsilon_{1} \varepsilon_{2} H_{0} w, \quad x_{1}=y_{1}+\varepsilon_{2} H_{1} w, \quad x_{2}=w+P_{0} x_{0}+P_{1} x_{1},
$$

where $H_{0}, H_{1}, P_{0}, P_{1}$ are matrix functions of appropriate dimensions.

If matrices $P_{0}$ and $P_{1}$ are solutions of the system

$$
\left\{\begin{array}{l}
\varepsilon_{1} \varepsilon_{2} \dot{P}_{0}=A_{20}+A_{22} P_{0}-\varepsilon_{1} \varepsilon_{2} P_{0} A_{00}-\varepsilon_{1} \varepsilon_{2} P_{0} A_{02} P_{0}-\varepsilon_{2} P_{1} A_{10}-\varepsilon_{2} P_{1} A_{12} P_{0}, \\
\varepsilon_{1} \varepsilon_{2} \dot{P}_{1}=A_{21}+A_{22} P_{1}-\varepsilon_{1} \varepsilon_{2} P_{0} A_{01}-\varepsilon_{1} \varepsilon_{2} P_{0} A_{02} P_{1}-\varepsilon_{2} P_{1} A_{11}-\varepsilon_{2} P_{1} A_{12} P_{1},
\end{array}\right.
$$

then system (1) will have the following form

$$
\left\{\begin{array}{c}
\dot{y}_{0}=\left(A_{00}+A_{02} P_{0}\right) y_{0}+\left(A_{01}+A_{02} P_{1}\right) y_{1}+\left(\varepsilon_{1} \varepsilon_{2} A_{00} H_{0}+\varepsilon_{2} A_{01} H_{1}-\varepsilon_{1} \varepsilon_{2} \dot{H}_{0}\right. \\
\left.+A_{02}\left(E+\varepsilon_{1} \varepsilon_{2} P_{0} H_{0}+\varepsilon_{2} P_{1} H_{1}\right)-H_{0}\left(A_{22}-\varepsilon_{1} \varepsilon_{2} P_{0} A_{02}-\varepsilon_{2} P_{1} A_{12}\right)\right) w \\
\varepsilon_{1} \dot{y}_{1}=\left(A_{10}+A_{12} P_{0}\right) y_{0}+\left(A_{11}+A_{12} P_{1}\right) y_{1}+\left(\varepsilon_{1} \varepsilon_{2} A_{10} H_{0}+\varepsilon_{2} A_{11} H_{1}-\varepsilon_{1} \varepsilon_{2} \dot{H}_{1}\right. \\
\left.\quad+A_{12}\left(E+\varepsilon_{1} \varepsilon_{2} P_{0} H_{0}+\varepsilon_{2} P_{1} H_{1}\right)-H_{1}\left(A_{22}-\varepsilon_{1} \varepsilon_{2} P_{0} A_{02}-\varepsilon_{2} P_{1} A_{12}\right)\right) w \\
\varepsilon_{1} \varepsilon_{2} \dot{w}=\left(A_{22}-\varepsilon_{1} \varepsilon_{2} P_{0} A_{02}-\varepsilon_{2} P_{1} A_{12}\right) w .
\end{array}\right.
$$


If matrices $H_{0}$ and $H_{1}$ are solutions of the system

$$
\left\{\begin{aligned}
\varepsilon_{1} \varepsilon_{2} \dot{H}_{0}= & \varepsilon_{1} \varepsilon_{2} A_{00} H_{0}+A_{02}\left(E+\varepsilon_{1} \varepsilon_{2} P_{0} H_{0}+\varepsilon_{2} P_{1} H_{1}\right) \\
& -H_{0}\left(A_{22}-\varepsilon_{1} \varepsilon_{2} P_{0} A_{02}-\varepsilon_{2} P_{1} A_{12}\right)+\varepsilon_{2} A_{01} H_{1}, \\
\varepsilon_{1} \varepsilon_{2} \dot{H}_{1}= & \varepsilon_{1} \varepsilon_{2} A_{10} H_{0}+A_{12}\left(E+\varepsilon_{1} \varepsilon_{2} P_{0} H_{0}+\varepsilon_{2} P_{1} H_{1}\right) \\
& -H_{1}\left(A_{22}-\varepsilon_{1} \varepsilon_{2} P_{0} A_{02}-\varepsilon_{2} P_{1} A_{12}\right)+\varepsilon_{2} A_{11} H_{1}
\end{aligned}\right.
$$

then system (4) will have the form

$$
\left\{\begin{array}{l}
\dot{y}_{0}=B_{00} y_{0}+B_{01} y_{1} \\
\varepsilon_{1} \dot{y}_{1}=B_{10} y_{0}+B_{11} y_{1} \\
\varepsilon_{1} \varepsilon_{2} \dot{w}=B_{22} w
\end{array}\right.
$$

where $B_{i j}=A_{i j}+A_{i 2} P_{j}, i, j=0,1, B_{22}=A_{22}-\varepsilon_{1} \varepsilon_{2} P_{0} A_{02}-\varepsilon_{2} P_{1} A_{12}$.

Under conditions C.1), C.2) and a sufficiently small value of the small parameter $\varepsilon_{2}>0$, in [8] it is established the existence of bounded solutions of systems (3) and (5) by which system (1) is reduced to the form (6).

Suppose that for the system (1) we should solve a Cauchy problem with initial conditions $\left(t_{0}, x_{00}, x_{10}, x_{20}\right)$. Let us show that there is a solution of the system (6) with initial conditions $\left(t_{0}, y_{00}, y_{10}, w_{0}\right)$ for which the equalities (2) are true. By the uniqueness of the solution, it suffices to show that (2) holds for $t=t_{0}$, that is

$$
\begin{aligned}
& x_{00}=y_{00}+\varepsilon_{1} \varepsilon_{2} H_{0}^{0} w_{0} \\
& x_{10}=y_{10}+\varepsilon_{2} H_{1}^{0} w_{0} \\
& x_{20}=w_{0}+P_{0}^{0} x_{00}+P_{1}^{0} x_{10} .
\end{aligned}
$$

From (7) we can get

$$
\begin{aligned}
& w_{0}=x_{20}-P_{0}^{0} x_{00}-P_{1}^{0} x_{10} \\
& y_{10}=x_{10}-\varepsilon_{2} H_{1}^{0}\left(x_{20}-P_{0}^{0} x_{00}-P_{1}^{0} x_{10}\right), \\
& y_{00}=x_{00}-\varepsilon_{1} \varepsilon_{2} H_{0}^{0}\left(x_{20}-P_{0}^{0} x_{00}-P_{1}^{0} x_{10}\right) .
\end{aligned}
$$

Therefore, each solution of the system (1) can be represented as (2), where $y_{0}, y_{1}, w$ is the solution of the system (6) with initial conditions (8).

Let us present this solution as

$$
\begin{aligned}
& x_{0}=x_{0}\left(t, \varepsilon_{1}, \varepsilon_{2}\right)=y_{0}\left(t, \varepsilon_{1}, \varepsilon_{2}\right)+\varphi_{1}\left(t, \varepsilon_{1}, \varepsilon_{2}\right), \\
& x_{1}=x_{1}\left(t, \varepsilon_{1}, \varepsilon_{2}\right)=y_{1}\left(t, \varepsilon_{1}, \varepsilon_{2}\right)+\varphi_{2}\left(t, \varepsilon_{1}, \varepsilon_{2}\right), \\
& x_{2}=x_{2}\left(t, \varepsilon_{1}, \varepsilon_{2}\right)=P_{0} y_{0}\left(t, \varepsilon_{1}, \varepsilon_{2}\right)+P_{1} y_{1}\left(t, \varepsilon_{1}, \varepsilon_{2}\right)+\varphi_{3}\left(t, \varepsilon_{1}, \varepsilon_{2}\right),
\end{aligned}
$$

where

$\varphi_{1}\left(t, \varepsilon_{1}, \varepsilon_{2}\right)=\varepsilon_{1} \varepsilon_{2} H_{0}^{0} w_{0}, \varphi_{2}\left(t, \varepsilon_{1}, \varepsilon_{2}\right)=\varepsilon_{2} H_{1}^{0} w_{0}, \varphi_{3}\left(t, \varepsilon_{1}, \varepsilon_{2}\right)=w+P_{0} \varphi_{1}\left(t, \varepsilon_{1}, \varepsilon_{2}\right)+P_{1} \varphi_{2}\left(t, \varepsilon_{1}, \varepsilon_{2}\right)$.

\section{Splitting and reduction principle}

Denote $Q\left(t, s, \varepsilon_{1}, \varepsilon_{2}\right)$ the fundamental matrix of the equation $\varepsilon_{1} \varepsilon_{2} \dot{x}_{2}=A_{22} x_{2}$.

The uniform boundedness of the matrix $A_{22}$ in the domain $\Omega$ and condition C.2) provide the estimate (see $[9,11,12])$

$$
\left\|Q\left(t, s, \varepsilon_{1}, \varepsilon_{2}\right)\right\| \leq K e^{-\frac{3 \beta}{2 \varepsilon_{1} \varepsilon_{2}}(t-s)}
$$

for some $K>0$ and any $-\infty<s \leq t<\infty$. 
Lemma 1. Let conditions C.1), C.2) be true. Then there exists $\overline{\varepsilon_{2}}$ such that for $0<\varepsilon_{2} \leq \overline{\varepsilon_{2}}$ the fundamental matrix $\bar{Q}\left(t, s, \varepsilon_{1}, \varepsilon_{2}\right)$ of the equation

$$
\varepsilon_{1} \varepsilon_{2} \dot{w}=\left(A_{22}-\varepsilon_{1} \varepsilon_{2} P_{0} A_{02}-\varepsilon_{2} P_{1} A_{12}\right) w
$$

satisfies the estimate

$$
\left|\bar{Q}\left(t, s, \varepsilon_{1}, \varepsilon_{2}\right)\right| \leq K e^{-\frac{\beta}{\varepsilon_{1} \varepsilon_{2}}(t-s)} .
$$

Proof. Rewrite the equation (11) as

$$
\varepsilon_{1} \varepsilon_{2} \dot{w}=A_{22} w-\left(\varepsilon_{1} \varepsilon_{2} P_{0} A_{02}+\varepsilon_{2} P_{1} A_{12}\right) w .
$$

The fundamental matrix $\bar{Q}\left(t, s, \varepsilon_{1}, \varepsilon_{2}\right)$ satisfies the integral equation

$$
\bar{Q}=Q-\int_{t_{0}}^{t} Q\left(\varepsilon_{1} \varepsilon_{2} P_{0} A_{02}+\varepsilon_{2} P_{1} A_{12}\right) \bar{Q} d s .
$$

Using estimation (10), condition C.1) and inequalities for matrices $\left\|P_{0}\right\| \leq \frac{K M}{\beta},\left\|P_{1}\right\| \leq \frac{K M}{\beta}$ (see [8]), we have

$$
|\bar{Q}| \leq K e^{-\frac{3 \beta}{2 \varepsilon_{1} \varepsilon_{2}}(t-s)}+\int_{t_{0}}^{t} \frac{K^{2} M^{2}}{\beta}\left(\varepsilon_{1} \varepsilon_{2}+\varepsilon_{2}\right)|\bar{Q}| e^{-\frac{3 \beta(t-s)}{2 \varepsilon_{1} \varepsilon_{2}}} d s .
$$

Applying the Gronwall-Bellman inequality, we obtain

$$
|\bar{Q}| e^{\frac{3 \beta t}{2 \varepsilon_{1} \varepsilon_{2}}} \leq K_{1} e^{\int_{t_{0}}^{t} \frac{K^{2} M^{2}}{\beta}\left(\varepsilon_{1} \varepsilon_{2}+\varepsilon_{2}\right) d s}=K_{1} e^{\frac{K^{2} M^{2}}{\beta}\left(\varepsilon_{1} \varepsilon_{2}+\varepsilon_{2}\right)\left(t-t_{0}\right)} .
$$

Therefore, $|\bar{Q}| \leq K e^{\left(\frac{K^{2} M^{2}}{\beta}\left(\varepsilon_{1} \varepsilon_{2}+\varepsilon_{2}\right)-\frac{3 \beta}{2 \varepsilon_{1} \varepsilon_{2}}\right) t}$. The last inequality for $\varepsilon<\frac{\beta}{\sqrt{2 \varepsilon_{2}\left(\varepsilon_{1}+1\right)} K M}=\overline{\varepsilon_{2}}$ takes the form

$$
|\bar{Q}| \leq K e^{-\frac{\beta}{\varepsilon_{1} \varepsilon_{2}}(t-s)}
$$

From the boundedness of the matrices $H_{0}, H_{1}$ and the estimate (12) we obtain that there is such positive number $N$ that for $t \geq t_{0}$ the following inequalities hold

$$
\begin{aligned}
& \left|\varphi_{1}\left(t, \varepsilon_{1}, \varepsilon_{2}\right)\right| \leq \varepsilon_{1} \varepsilon_{2} N e^{-\frac{\beta}{\varepsilon_{1} \varepsilon_{2}}\left(t-t_{0}\right)}\left|w_{0}\right|, \\
& \left|\varphi_{2}\left(t, \varepsilon_{1}, \varepsilon_{2}\right)\right| \leq \varepsilon_{2} N e^{-\frac{\beta}{\varepsilon_{1} \varepsilon_{2}}\left(t-t_{0}\right)}\left|w_{0}\right|, \\
& \left|\varphi_{3}\left(t, \varepsilon_{1}, \varepsilon_{2}\right)\right| \leq N e^{-\frac{\beta}{\varepsilon_{1} \varepsilon_{2}}\left(t-t_{0}\right)}\left|w_{0}\right| .
\end{aligned}
$$

From the obtained relations (9) and estimates (13) we obtain that the system (1) has the integral manifold

$$
x_{2}=P_{0} x_{0}+P_{1} x_{1}
$$

the motion on which describes the subsystem of the first two equations of the system (6)

$$
\left\{\begin{array}{l}
\dot{y}_{0}=B_{00} y_{0}+B_{01} y_{1} \\
\varepsilon_{1} \dot{y}_{1}=B_{10} y_{0}+B_{11} y_{1} .
\end{array}\right.
$$

Any solution of system (1) is represented as the sum of some solution that lies on the integral manifold and exponentially decreasing terms. From the representation (9) and the estimates (13) we obtain statements for the investigation of the stability of the zero solution of system (1). 
Theorem 1. Let the conditions C.1), C.2) be true. Then for sufficiently small $\varepsilon_{1}, \varepsilon_{2}$ the zero solution of system (1) is stable (asymptotically stable, unstable) if and only if the zero solution of subsystem (14) is stable (asymptotically stable, unstable).

Let us now consider system (6), which has already been studied in $[9,10]$. Let the following condition be true

C.3) eigenvalues $\lambda_{i}=\lambda_{i}(t), i=\overline{0, n_{1}}$, of the matrix $B_{11}\left(t, \varepsilon_{1}, \varepsilon_{2}\right)$ satisfy the inequality

$$
\operatorname{Re} \lambda_{i} \leq-2 \gamma<0
$$

Then there exists $\varepsilon_{1}^{*}>0$ such that for $0<\varepsilon_{1}<\varepsilon_{1}^{*}$ the substitution of variables (see [8,9])

$$
\left\{\begin{array}{l}
y_{0}=u+\varepsilon_{1} H\left(t, \varepsilon_{1}, \varepsilon_{2}\right) v \\
y_{1}=v+P\left(t, \varepsilon_{1}, \varepsilon_{2}\right) y_{0}
\end{array}\right.
$$

splits the system (6) into independent subsystems

$$
\left\{\begin{array}{l}
\dot{u}=\left(B_{00}+B_{01}\right) H u \\
\varepsilon_{1} \dot{v}=\left(B_{11}-\varepsilon_{1} H B_{01}\right) v .
\end{array}\right.
$$

The matrix functions $P$ and $H$ are uniformly bounded solutions of such equations

$$
\left\{\begin{array}{l}
\varepsilon_{1} \dot{P}=\varepsilon_{1}\left(B_{00}+B_{01} H\right) P+B_{01}-P\left(B_{11}-\varepsilon_{1} H B_{01}\right) \\
\varepsilon_{1} \dot{H}=B_{10}+B_{11} H-\varepsilon_{1} H\left(B_{00}-B_{01} H\right)
\end{array}\right.
$$

By expressing the old variables $x_{0}, x_{1}, x_{2}$ through the new ones $u, v, w$ we obtain the following result.

Theorem 2 ([8]). Let conditions C.1), C.2), C.3) be satisfied. Then for sufficiently small values $\varepsilon_{1}, \varepsilon_{2}$, there is a nondegenerate substitution of variables

$$
\left(\begin{array}{c}
x_{0} \\
x_{1} \\
x_{2}
\end{array}\right)=\left(\begin{array}{ccc}
E & \varepsilon_{1} H & \varepsilon_{1} \varepsilon_{2} H_{0} \\
P & E+\varepsilon_{1} P H & \varepsilon_{2} H_{1} \\
P_{0}+P_{1} P & P_{1}+\varepsilon_{1}\left(P_{0}+P_{1} P\right) H & E+\varepsilon_{1} \varepsilon_{2} P_{0} H_{0}+\varepsilon_{2} P_{1} H_{1}
\end{array}\right)\left(\begin{array}{l}
u \\
v \\
w
\end{array}\right),
$$

by which system (1) is transformed to three independent subsystems

$$
\left\{\begin{array}{l}
\dot{u}=\left(B_{00}+B_{01} H\right) u \\
\varepsilon_{1} \dot{v}=\left(B_{11}-\varepsilon_{1} H B_{01}\right) v \\
\varepsilon_{1} \varepsilon_{2} \dot{w}=B_{22} w
\end{array}\right.
$$

In system (16) the first equation describes the behavior of system (6) on the invariant manifold of slow variables, and the second equation provides the behavior of system (6) on the invariant manifold of fast variables.

From representation (15) under condition C.3), it was established in $[9,10]$ that the stability of system (6) is equivalent to the stability of the first equation of system (16).

Let us summarize these considerations in the form of reduction principle for investigating the stability of the zero solution of the original system (1). 
Theorem 3. Let conditions C.1), C.2), C.3) be satisfied. Then for sufficiently small values $\varepsilon_{1}, \varepsilon_{2}$ the zero solution of system (1) is stable (asymptotically stable, unstable) if and only if the zero solution of equation

$$
u^{\prime}(t)=\left(B_{00}+B_{01} H\right) u
$$

is stable (asymptotically stable, unstable).

Example. As an example of application of construction of splitting transformation and application of the principle of reduction we will consider system of singularly perturbed differential equations

$$
\left\{\begin{array}{l}
\dot{x_{0}}=x_{0}+x_{1}+x_{2}, \\
\varepsilon_{1} \dot{x_{1}}=x_{0}+x_{1}+x_{2}, \\
\varepsilon_{1} \varepsilon_{2} \dot{x_{2}}=-x_{0}-x_{1}-x_{2} .
\end{array}\right.
$$

Let us make in (17) the substitution

$$
x_{0}=y_{0}+\varepsilon_{1} \varepsilon_{2} h_{0} w, \quad x_{1}=y_{1}+\varepsilon_{2} h_{1} w, \quad x_{2}=w+p_{0} x_{0}+p_{1} x_{1} .
$$

To find the coefficients $p_{0}, p_{1}, h_{0}, h_{1}$, taking into account the stationarity and form of the system (17) from relations (3) and (5) we obtain the following systems of equations:

$$
\begin{gathered}
\left\{\begin{array}{c}
-1-p_{0}-\varepsilon_{1} \varepsilon_{2} p_{0}-\varepsilon_{1} \varepsilon_{2} p_{0}^{2}-\varepsilon_{2} p_{1}-\varepsilon_{2} p_{0} p_{1}=0, \\
-1-p_{1}-\varepsilon_{1} \varepsilon_{2} p_{0}-\varepsilon_{1} \varepsilon_{2} p_{0} p_{1}-\varepsilon_{2} p_{1}-\varepsilon_{2} p_{1}^{2}=0,
\end{array}\right. \\
\left\{\begin{array}{l}
h_{0}\left(-1+\varepsilon_{1} \varepsilon_{2}+\varepsilon_{2}\right)=1 \\
h_{1}\left(-1+\varepsilon_{1} \varepsilon_{2}+\varepsilon_{2}\right)=1 .
\end{array}\right.
\end{gathered}
$$

Finding the bounded solutions of these systems, we get

$$
p_{0}=p_{1}=-1, \quad h_{0}=h_{1}=\frac{1}{-1+\varepsilon_{1} \varepsilon_{2}+\varepsilon_{2}} .
$$

Thus, the substitution of variables (18) takes the form

$$
x_{0}=y_{0}+\frac{\varepsilon_{1} \varepsilon_{2}}{-1+\varepsilon_{1} \varepsilon_{2}+\varepsilon_{2}} w, \quad x_{1}=y_{1}+\frac{\varepsilon_{2}}{-1+\varepsilon_{1} \varepsilon_{2}+\varepsilon_{2}} w, \quad x_{2}=w-x_{0}-x_{1} .
$$

In this case, the system (17) is split into the system of independent equations

$$
\left\{\begin{array}{l}
\dot{y_{0}}=0, \\
\varepsilon_{1} \dot{y}_{1}=0 \\
\varepsilon_{1} \varepsilon_{2} \dot{w}=\left(-1+\varepsilon_{1} \varepsilon_{2}+\varepsilon_{2}\right) w .
\end{array}\right.
$$

According to Theorem 3, the stability of the zero solution of system (17) is equivalent to the stability of the zero solution of the first equation of system (19). Thus, based on the principle of reduction, we can conclude that the zero solution of system (17) is stable, but not asymptotic.

\section{References}

[1] Cherevko I.M., Osypova O.V. Asymptotic decomposition of linear singularly perturbed multiscale systems. Miskolc Math. Notes 2015, 16 (2), 729-745. doi:10.18514/MMN.2015.1627

[2] Cherevko I.M. Splittin of linear singularly perturbed differential-functional equations. Dopov. Nats. Akad. Nauk Ukr. 2002, 6, 32-36. (in Ukrainian) 
[3] Gol'dshteyn N.V., Sobolev V.A. Qualitative analysis of singularly perturbed systems. Institute Math. Publ., Novosibirsk, 1988. (in Russian)

[4] Mitropol'skiy Yu.A., Lykova O.B. Integral manifolds in non-linear mechanics. Nauka, Moscow, 2013. (in Russian)

[5] Osypova O.V., Cherevko I.M. On splitting and decomposition of linear stationary singularly perturbed differential equations. Bukovinian Math. J. 2019, 7 (2), 76-85. (in Ukrainian).

[6] Sakamoto K. Invariant manifolds in singular perturbation problems for ordinary differential equations. Proc. Roy. Soc. Edinburgh Sect. A 1990, 116 (1-2), 45-78. doi:10.1017/S0308210500031371

[7] Schepakina E.A., Sobolev V.A., Mortell M.P. Singular Perturbations: Introduction to system order reduction methods with applications. Springer, Berlin, 2014.

[8] Sel's'kyy S.S., Cherevko I.M. Integral manifolds and decomposition of the systems of linear singularly perturbed equations with two small parameters. Visnyk of the Chernivtsi Univ. Ser. Matematyka 2011, 1 (3), 104-107. (in Ukrainian)

[9] Sobolev V.A. Decomposition of linear singularly perturbed systems. Acta Math. Hung. 1987, 4 (3-4), $365-376$. doi:10.1007/BF01950998

[10] Sobolev V.A. Integral manifolds and decomposition of singularly perturbed system. Systems Control Lett. 1984, 5 (3), 169-179. doi:10.1016/S0167-6911(84)80099-7

[11] Strygin V.V., Sobolev V.A. Separation of motions by the method of integral manifolds. Nauka, Moscow, 1988. (in Russian)

[12] Voropaeva N.V., Sobolev V.A. Geometrical decomposition of singularly perturbed systems. Fizmatlit, Moscow, 2009. (in Russian)

Received 12.05.2020

Осипова О.В., Перцов А.С., Черевко І.М. Аекомпозиція та стійкість лінійних сингулярно збурених систем з двома малими параметрами // Карпатські матем. публ. - 2021. - Т.13, №1. - С. 15-21.

В області $\Omega=\left\{\left(t, \varepsilon_{1}, \varepsilon_{2}\right): t \in \mathbb{R}, \varepsilon_{1}>0, \varepsilon_{2}>0\right\}$ досліджується лінійна сингулярно збурена система з двома малими параметрами

$$
\left\{\begin{array}{l}
\dot{x}_{0}=A_{00} x_{0}+A_{01} x_{1}+A_{02} x_{2} \\
\varepsilon_{1} \dot{x}_{1}=A_{10} x_{0}+A_{11} x_{1}+A_{12} x_{2} \\
\varepsilon_{1} \varepsilon_{2} \dot{x}_{2}=A_{20} x_{0}+A_{21} x_{1}+A_{22} x_{2}
\end{array}\right.
$$

де $x_{0} \in \mathbb{R}^{n_{0}}, x_{1} \in \mathbb{R}^{n_{1}}, x_{2} \in \mathbb{R}^{n_{2}}$. Розглянуто схеми декомпозиціі та розщеплення системи на незалежні підсистеми за допомогою інтегральних многовидів швидких та повільних змінних. Встановлено умови, при виконанні яких справедливий принцип зведення для дослідження стійкості нульового розв' язку вихідної системи.

Ключові слова і фрази: сингулярно збурена система, декомпозищія, розщеплення, стійкість, інтегральний многовид. 